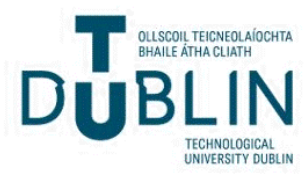

Technological University Dublin

ARROW@TU Dublin

\section{A Vibrational Spectroscopic Based Approach for Diagnosing Babesia Bovis Infection}

Anja Ruther

Monash University

David Perezguaita

Technological University Dublin, david.perezguaita@tudublin.ie

William Poole

Monash University

See next page for additional authors

Follow this and additional works at: https://arrow.tudublin.ie/scschphyart

Part of the Atomic, Molecular and Optical Physics Commons, and the Biological and Chemical Physics Commons

\section{Recommended Citation}

Ruther, A. et al. (2020) A vibrational spectroscopic based approach for diagnosing Babesia bovis infection, Analytical Chemistry, XXXX(XXX), June 2020. DOI: 10.1021/acs.analchem.0c00150

This Article is brought to you for free and open access by the School of Physics \& Clinical \& Optometric Science at ARROW@TU Dublin. It has been accepted for inclusion in Articles by an authorized administrator of ARROW@TU Dublin. For more information, please contact arrow.admin@tudublin.ie, aisling.coyne@tudublin.ie, gerard.connolly@tudublin.ie.

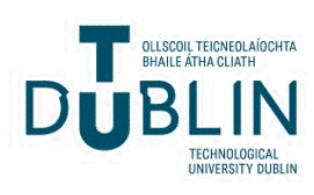




\section{Authors}

Anja Ruther, David Perezguaita, William Poole, Brian Cooke, Carlos Suarez, Philip Heraud, and Bayden Wood 


\section{Article}

\section{A vibrational spectroscopic based approach for diagnosing Babesia bovis infection}

Anja Rüther, David Perez-Guaita, William A. Poole, Brian M.

Cooke, Carlos E. Suarez, Philip Heraud, and Bayden R. Wood

Anal. Chem., Just Accepted Manuscript • DOI: 10.1021/acs.analchem.0c00150 • Publication Date (Web): 01 Jun 2020

Downloaded from pubs.acs.org on June 2, 2020

\section{Just Accepted}

"Just Accepted" manuscripts have been peer-reviewed and accepted for publication. They are posted online prior to technical editing, formatting for publication and author proofing. The American Chemical Society provides "Just Accepted" as a service to the research community to expedite the dissemination of scientific material as soon as possible after acceptance. "Just Accepted" manuscripts appear in full in PDF format accompanied by an HTML abstract. "Just Accepted" manuscripts have been fully peer reviewed, but should not be considered the official version of record. They are citable by the Digital Object Identifier (DOI®). "Just Accepted" is an optional service offered to authors. Therefore, the "Just Accepted" Web site may not include all articles that will be published in the journal. After a manuscript is technically edited and formatted, it will be removed from the "Just Accepted" Web site and published as an ASAP article. Note that technical editing may introduce minor changes to the manuscript text and/or graphics which could affect content, and all legal disclaimers and ethical guidelines that apply to the journal pertain. ACS cannot be held responsible for errors or consequences arising from the use of information contained in these "Just Accepted" manuscripts. 


\title{
A vibrational spectroscopic based approach for diagnosing Babesia bovis infection
}

Anja Rüther ${ }^{1}$, David Perez-Guaita ${ }^{1}$, William A. Poole ${ }^{2}$, Brian M. Cooke ${ }^{2}$, Carlos E. Suarez ${ }^{3}$, Philip Heraud, ${ }^{1,2 *}$, Bayden R. Wood ${ }^{1 *}$

${ }^{1}$ Centre for Biospectroscopy, School of Chemistry, Monash University, Wellington Rd, Clayton Campus, VIC 3800, Australia.

2Department of Microbiology, Biomedicine Discovery Institute, Monash University, Wellington Rd, Clayton Campus, VIC 3800, Australia

${ }^{3}$ Department of Veterinary Microbiology and Pathology, Washington State University, Pullman, Washington, USA.

*To whom correspondence should be addressed (Bayden.wood@monash.edu, Phil.Heraud@monash.edu)

\begin{abstract}
Babesia bovis parasites present a serious and significant health concern for the beef and dairy industries in many parts of the world. Difficulties associated with the current diagnostic techniques include they are prone to human error (microscopy) or expensive and time consuming (Polymerase Chain Reaction) to perform. Little is known about the biochemical changes in blood that are associated with Babesia infections. The discovery of new biomarkers will lead to improved diagnostic outcomes for the cattle industry. Vibrational spectroscopic technologies can record a chemical snapshot of the entire organism and the surrounding cell thereby providing a phenotype of the organism and the host infected cell. Here, we demonstrate the applicability of vibrational spectroscopic imaging techniques including Atomic Force Microscopy Infrared (AFM-IR) and confocal Raman microscopy to discover new biomarkers for B. bovis infections. Furthermore, we applied Attenuated Total Reflection Fourier Transform Infrared (ATR-FTIR) to detect B. bovis in red blood cells (RBCs). Based on changes in the IR spectral bands, ATR-FTIR in combination with Partial
\end{abstract}


Least Squares-Discriminant Analysis we were able to discriminate infected samples from controls with a sensitivity and specificity of $92.0 \%$ and $91.7 \%$, respectively in less than two minutes, excluding sample extraction and preparation. The proposed method utilized a lysis approach to remove hemoglobin from the suspension of infected and uninfected cells, which significantly increased the sensitivity and specificity compared to measurements performed on intact infected red blood cells (intact infected RBC, 77.3\% and 79.2\%). This work represents a holistic spectroscopic study from the level of the single infected RBC using AFM-IR and confocal Raman to the detection of the parasite in a cell population using ATR-FTIR for a babesiosis diagnostic.

KEYWORDS: Babesiosis, Vibrational Spectroscopy, Imaging, Diagnosis

\section{Introduction}

Tick-borne Babesia parasites are responsible for important diseases in humans and animals globally. Babesiosis has a large impact on human health in some countries, but it also has devastating effects on the cattle industry worldwide, and improved diagnostic and control measures are urgently needed. Bovine babesiosis (caused predominantly by B. bovis and B. bigemina) is an important tickborne disease that affects animals in many regions of the world, including South- and Middle America, Africa, Asia and Australia. Bovine babesiosis manifests as fever, anorexia and anemia, and in its most severe form, cerebral babesiosis, which is frequently fatal. The economic impact of bovine babesiosis is severe in the many countries affected by the disease largely due to its negative effects on the productivity of the beef and dairy industries. ${ }^{1,2}$ The reintroduction of $B$. bovis from Middle and South America into the USA is a constant threat, especially with increasing climate change and increasing globalization, which therefore warrants the development of better diagnostic/detection methods. ${ }^{3}$ Babesiosis is often transmitted simultaneously with other tick- 
borne diseases and can show similar clinical symptoms to anaplasmosis or theileriosis and hence the estimated number of undetected cases remains high., ${ }^{4,5}$

Bovine babesiosis can be diagnosed currently using direct blood inspection using microscopy, and serological (IFAT, ELISA) methods but all of these methods have limitations. Thus, the gold standard in diagnostic methods for babesiosis include microscopy and polymerase chain reaction (PCR) . Microscopy is time consuming, and does not offer high sensitivity due to low parasitemia in babesiosis, and misdiagnosis is often a problem. In addition, microscopy is unable to discriminate between $B$. bovis and $B$. bigemina infections. In contrast, PCR methods are highly sensitive and specific, but time consuming, cumbersome, and expensive. Serological testing such as indirect fluorescent antibody test (IFAT) and the enzyme-linked immunosorbent assay (ELISA) require laboratory equipment and chemicals. Additionally, serological methods are mainly applied for research purposes and epidemiological studies, for export certification or when vaccine breakdowns are suspected and are not suitable for point-of-care diagnosis. ${ }^{6}$ Here, we propose an alternative diagnostic method, using vibrational spectroscopy probing the absorbance of infrared (IR) light or Raman scattering, both leading to molecular vibrations in a sample. ${ }^{7}$ Biological samples show characteristic spectral patterns, correlating to the absorbance or scattering of light, e.g., from lipids, proteins, carbohydrates and nucleic acids. Changes in the biochemical composition of a blood sample infected with a parasite can consequently be detected using vibrational spectroscopy. A number of vibrational spectroscopic methods in combination with chemometric data analysis have been explored for various biomedical purposes. ${ }^{8}$ IR and Raman based microspectroscopic imaging techniques such as confocal Raman imaging and Focal Plane Array (FPA) IR imaging allow for establishing local characteristics in individual infected red blood cells (iRBCs) and can be integrated in a multimodal approach to receive information about the spatial position of molecules within cells. ${ }^{9}$ In theory, IR and Raman provide complementary information of the parasite-host system. 
Lipids, proteins, carbohydrates and DNA found in the parasite dominate the FTIR spectrum, whereas in the case of Raman, heme modes from hemoglobin are enhanced due to the resonance effect. Raman microscopy that utilizes excitation in the visible range enables images to be collected with a lateral spatial resolution approaching $0.3 \mu \mathrm{m}$, depending on the laser wavelength and optical configuration. The lateral spatial resolution of IR imaging techniques is usually limited by the refractive index of the incident light beam to 2 to $10 \mu \mathrm{m}$. This drawback can be overcome by coupling FTIR spectroscopy to Atomic Force Microscopy (AFM). AFM-IR allows for the collection of IR spectra with a nanoscale spatial resolution, as a very fine tip records the response of the molecules to the IR light. To obtain IR spectra at defined positions on the sample, the tip has to be placed directly onto the target such as a parasite inside a red blood cell (RBC).

The aforementioned imaging techniques are very suitable for research applications (e.g. studies of the mode of drug action), ${ }^{10}$ but their use as a high throughput diagnostic tool is limited by the size and price of the instrumentation. In contrast, Attenuated Total Reflection (ATR) Fourier Transform (FT) IR spectroscopy records the overall chemical composition of a bulk sample. The technique uses compact instrumentation and is able to acquire a spectrum of an untreated sample within minutes, making it ideal for Point-of-Care (PoC) analysis. ATR-FTIR spectroscopy has for example been applied to the detection of other parasite related diseases such as malaria. ${ }^{11}$

Here, we demonstrate the applicability of vibrational spectroscopic methods for the detection of $B$. bovis in bovine RBCs. Due to complex and subtle differences in the spectra of iRBCs and uninfected RBCs (uRBCs) chemometric data analysis was performed to extract the spectral information that correspond to $B$. bovis parasite. By applying AFM-IR we could assign spectral features characteristic of the parasite measured directly inside RBCs, such as DNA and lipid IR-bands. Confocal Raman microscopy was further used to obtain images of the parasite inside the RBC. Every pixel $(0.3 \mu \mathrm{m} x$ 
$0.3 \mu \mathrm{m})$ in the image contains a Raman spectrum. The AFM-IR spectra were used to identify potential marker bands of $B$. bovis infection to ultimately develop a simpler ATR-FTIR spectroscopybased method combined with chemometric data analysis for the detection of B. bovis. Partial least squares analysis (PLS-DA) was used to determine sensitivity (true positives/false negatives) and specificity (true negatives/false positives) of the method. Spectroscopic methods are quick, easy and reliable for detecting disease-induced phenotypic changes through characteristic molecular vibrations. In addition, the use of spectroscopic methods can also probe a range of molecules at the same time rather than focusing on only on a few biomarkers, as is the case with most phenotypic methods such as immunohistochemistry, for example. This means that even complex biological samples can be unraveled based on their biochemical fingerprint. Thus, ATR-FTIR might serve as a PoC non-subjective diagnostic tool that allows for a differential diagnosis of one disease in presence of another without the pre-requisite knowledge of a tick bite. This study serves as a proof of concept to detect bovine babesiosis and might potentially be adapted to human babesiosis as a rapid screening tool to increase the safety of blood transfusions.

\section{Materials and methods}

In vitro culture of Babesia bovis and sample preparation

Babesia bovis parasites were maintained in continuous in vitro culture in bovine RBCs suspended in HEPES-buffered RPMI-1640 with L-glutamine medium supplemented with Albumax II (1\% w/v), hypoxanthine $(300 \mu \mathrm{M})$ and sodium bicarbonate $(2 \mathrm{~g} / \mathrm{L})$ as previously described. ${ }^{12}$ Parasites were cultured at $5 \%$ hematocrit in gassed $\left(1 \% \mathrm{O}_{2}, 5 \% \mathrm{CO}_{2}\right.$ and $\left.94 \% \mathrm{~N}_{2}\right)$ sealed tissue culture flasks at $37^{\circ} \mathrm{C}$. Cultures were diluted with fresh bovine RBCs approximately every 48 hours to maintain parasitemia below $10 \%$. Bovine RBCs used for culture were separated from serum, washed with Vega y Martinez Solution (VYMS) $)^{13}$ and stored at $4^{\circ} \mathrm{C}$. 
These cultured bovine RBCs infected with B. bovis (6.8\%-17.5\% parasitemia) were centrifuged (2000 $x g, 5 \mathrm{~min}$ ) and the supernatant (culture medium) was removed. The pellet (packed RBCs) was washed once with isotonic saline. Samples were then treated according to the specific needs of the technique (See following sections).

AFM-IR

AFM-IR spectra were recorded on the NanoIR2 instrument (Anasys Instruments, Santa Barbara, CA 93101, USA) using an optical parametric oscillator laser as a light source in the spectral range of 900 to $1800 \mathrm{~cm}^{-1}$. The output power of the laser was aligned with the expected signal intensities according to the following scheme:

\begin{tabular}{|c|c|}
\hline Wavelength & Laser \\
\hline region & power \\
\hline $900-1500 \mathrm{~cm}^{-1}$ & $19.4 \%$ \\
\hline $1500-1600 \mathrm{~cm}^{-1}$ & $15.25 \%$ \\
\hline $1600-1700 \mathrm{~cm}^{-1}$ & $11.42 \%$ \\
\hline $1700-1800 \mathrm{~cm}^{-1}$ & $19.4 \%$ \\
\hline
\end{tabular}

IR Amplitude spectra were recorded with $4 \mathrm{~cm}^{-1}$ spectral resolution.

IR maps were recorded with a scan rate of $0.07 \mathrm{kHz}$ with a resolution of 200 points. Multispectral IR images were obtained by collecting several maps at different wavenumbers (940, 970, 1010, 1055, $1079,1106,1130,1237,1353,1457,1550,1656,1720$ and $1740 \mathrm{~cm}^{-1}$ ) at the same area and overlaying them in a self-written MATLAB tool. Topographic images were recorded simultaneously to the collection of the IR maps. 


\section{Confocal Raman microscopy}

Hyperspectral Raman images were recorded using a confocal Raman microscope (WITec alpha300 $\mathrm{R}$, Melbourne, Australia) with an air-cooled solid-state laser operating at $532 \mathrm{~nm}$ as a light source with a grating of 600 grooves $/ \mathrm{mm}(B L Z=500 \mathrm{~nm})$ and a back-illuminated CCD camera, which was cooled to $-60{ }^{\circ} \mathrm{C}$. All measurements were controlled using the WITec Control Software and performed with a $100 \times$ dry objective (Olympus).

Before spectra collection, a calibration was performed using the Raman scattering line of silica plate $\left(520.5 \mathrm{~cm}^{-1}\right)$. The spectra were collected in the spectral range from 0 to $4000 \mathrm{~cm}^{-1}$ with a resolution of $3 \mathrm{~cm}^{-1}$, an integration time of $10 \mathrm{~s}$ and a sampling density of $0.1 \mathrm{~mm}$.

Samples were smeared on a $\mathrm{CaF}_{2}$ crystal, creating a monolayer of RBCs, which were fixed by dipping the crystal in a solution of methanol. Then the crystal was placed on the AFM-IR instrument, and after locating the cells using the visible objective, AFM and AFM-IR along with single point spectra were acquired. In total, 3 biological replicates were analyzed 5 cells were mapped and 150 spectra were obtained. Fore presentation we selected a representative image that clearly shows the parasite.

\section{ATR-FTIR}

ATR-FTIR measurements were carried out on a Bruker Alpha spectrometer (Bruker, Billerica, MA, USA), equipped with an ATR diamond accessory. It was set to a resolution of $6 \mathrm{~cm}^{-1}$ with 64 interferograms co-added per spectrum (background: 64 scans), a spectral range of $400-4000 \mathrm{~cm}^{-1}$ and a data interval of $2 \mathrm{~cm}^{-1}$ was selected. Three measurement replicates were performed per sample. Background measurements were taken before each replicate.

For ATR-FTIR spectroscopy, the iRBCs were diluted with uRBCs, which have been spun down and washed to obtain final parasitemia of $0.0001 \%, 0.001 \%, 0.01 \%, 0.1 \%, 0.25 \%, 0.5 \%, 1 \%, 1.5 \%, 2 \%$, and 3\%. Each mixture (12 mixtures per concentration) was washed again with isotonic saline. For 
samples of intact RBCs, three spots of each mixture were prepared on a glass slide using $5 \mu \mathrm{L}$ each and allowed to dry in air. The drying step caused a decrease in the water bands and an increase in bands associated to other biochemical compounds (proteins, lipids, carbohydrates and DNA). Samples were dried for a few hours until the contribution of water (bands at 3264 and $1632 \mathrm{~cm}^{-1}$ ) were negligible in the IR spectrum.

For RBC lysis, $350 \mu \mathrm{L}$ distilled water was added to $50 \mu \mathrm{L}$ of packed RBCs. The mixtures were shaken, centrifuged $(13,000 \times g, 5 \mathrm{~min})$ and the supernatant was removed. Four spots of each mixture were prepared on a glass slide using $7.5 \mu \mathrm{L}$ each and allowed to dry in the air.

\section{Data analysis}

Data were analyzed in MATLAB (MathWorks, Natick, MA) using the PLS toolbox and a lab-written script in MATLAB (Gplotter) for image analysis. Each spectrum was smoothed or converted into its first or second derivative (Savitzky-Golay filter, smoothing), normalized (standard normal variate, SNV, or multiplicative scatter correction, MSC), Pareto scaled if applicable and mean centered. Sensitivity and specificity were calculated using partial least squares discriminant analysis.

\section{Partial least squares discriminant analysis}

Partial least squares discriminant analysis (PLSDA) was performed using the PLS toolbox in MATLAB. Single point AFM-IR spectra were preprocessed (9 or 13 pt Savitzky-Golay smoothing (3 ${ }^{\text {rd }}$ order), SNV, mean centering) and spectral sets from three different AFM tip positions (on the parasite, on the body of the $\mathrm{iRBC}$ and on one $\mathrm{URBC}$ ) were discriminated. Spectra corresponding to pixels in hyperspectral Raman images were preprocessed (truncation of the region $<400 \mathrm{~cm}^{-1}$, smoothing 15 points Savitzky-Golay ( $2^{\text {nd }}$ order), SNV, mean centering) and discriminated based on their localization ("parasite" and "cytoplasm") on the cell. For ease of spectral interpretation, the second 
derivative was assessed where the maxima of the original spectra become minima in the second derivative.

All ATR-FTIR data were corrected for potential background absorbance from water vapor. For this purpose, an independently recorded spectrum of water vapor (normalized for the band between $3545.67 \mathrm{~cm}^{-1}$ and $3570.31 \mathrm{~cm}^{-1}$ ) was subtracted from the data. The pre-processed ATR-FTIR spectra of lysed samples (group 1: $0.001 \%$ to $0.1 \%$ parasitemia, group 2: $0.25 \%$ to $1 \%$ parasitemia) were analyzed over the three combined regions; the $\mathrm{CH}$ stretching region $\left(2980-2800 \mathrm{~cm}^{-1}\right)$, the ester carbonyl region $\left(1800-1700 \mathrm{~cm}^{-1}\right)$ and the carbohydrate region $\left(1450-900 \mathrm{~cm}^{-1}\right)$, while the spectra of intact RBCs (group 1: $0.01 \%$ to $0.1 \%$ parasitemia, group 2: $0.25 \%$ to $1 \%$, group $3: 1.5 \%$ to $3 \%$ parasitemia) were analyzed in the fingerprint region $\left(1800-900 \mathrm{~cm}^{-1}\right)$. All spectra were preprocessed as shown in Table 1 and discriminated based on B. bovis infection.

\section{Table 1}

\begin{tabular}{|c|c|c|c|}
\hline Sample & Preprocessing & Spectral regions $\left(\mathrm{cm}^{-1}\right)$ & Latent variables \\
\hline Lysed $0.25 \%-1 \%$ & 1st Der. (23 pt.), MSC, & $3042.75-2804.59$ & 6 \\
\hline & PS, MC & 1784.18-1704.11 & \\
\hline & & 1531.64-1016.3 & \\
\hline $0.001 \%-$ & 2nd Der. (23 pt), SNV, PS, & $3044.29-2804.11$ & 4 \\
\hline $0.1 \%$ & MC & 1792.09-1699.71 & \\
\hline & & 1533.43-956.6 & \\
\hline Intact RBCs & 1st Der. (23 pt,), SNV, MC & $1802.61-1016.28$ & 4 \\
\hline
\end{tabular}




$\begin{array}{ll}\begin{array}{l}1.5 \%-3 \% \\ \text { Intact RBCs }\end{array} & \text { 1st Der. (23 pt,), MSC, 1782.08-1001.9 } \\ 0.25 \%-1 \% & \text { MC } \\ \text { Intact RBCs } & \text { 1st Der. (19 pt), SNV, PS, 1802.66-948.55 } \\ 0.01 \%-0.1 \% & M C\end{array}$

\section{Cluster Analysis}

k-Means Cluster Analysis (kMCA) was performed using a lab-written tool in MATLAB (Gplotter) on the preprocessed (SNV) multispectral AFM-IR image (included wavenumber values: 940, 970, 1010, $1055,1079,1106,1130,1237,1353,1457,1550,1656,1720$ and $1740 \mathrm{~cm}^{-1}$ ) and on the preprocessed (truncation of the region $<400 \mathrm{~cm}^{-1}$, first derivative, 15 points Savitzky-Golay smoothing ( $1^{\text {st }}$ order), mean centering) on the hyperspectral Raman image.

\section{Results \\ Phenotypic studies of Babesia bovis-infected RBCs \\ AFM-IR spectroscopy \\ A topographical map obtained by AFM (Figure 1A), was recorded before IR spectra were collected, showed two collapsed depressions in the iRBC (iRBC) that are indicative of the parasite in the RBC. ${ }^{14}$ The same type of depression in the RBC was observed in Plasmodium spp-infected human RBCs using AFM-IR and was explained by a destruction of the RBC cytoskeleton with a simultaneous increase in mesh size of the cytoskeletal network during schizogony of the parasite. ${ }^{15}$ The AFM probe tip was positioned directly on the region of the iRBC in direct contact with the intracellular parasite, on the cytoplasm of the $\mathrm{iRBC}$, and on another $\mathrm{URBC}$ and spectra were recorded (Figure 1A).}


Figure $1 B$ shows the averaged spectra $(n=26-27)$ from different tip positions. The most distinct differences were observed in the averaged spectra are between uRBCs and iRBCs, while spectra acquired from the cytoplasm of the $\mathrm{iRBC}$ and on the parasite containing region spectra appear similar. The amide I band, which is associated with mainly $\mathrm{C}=\mathrm{O}$ stretching modes of the peptide group in proteins ${ }^{16}$ seems to be slightly more intense in the spectra from the cytoplasm surrounding the infected cells, while it is the weakest where the parasite is actually located. ${ }^{16}$

In an IR spectrum, the phenotype is represented as a complex set of overlapped bands, making necessary the use of multivariate data analysis to extract the biochemical information. ${ }^{17}$ Two PLSDA models (Figure 1C) were developed: one discriminating the parasite within the cell from the surrounding iRBC cytoplasm (model 1); the other between iRBC cytoplasm and the uRBC (model 2).
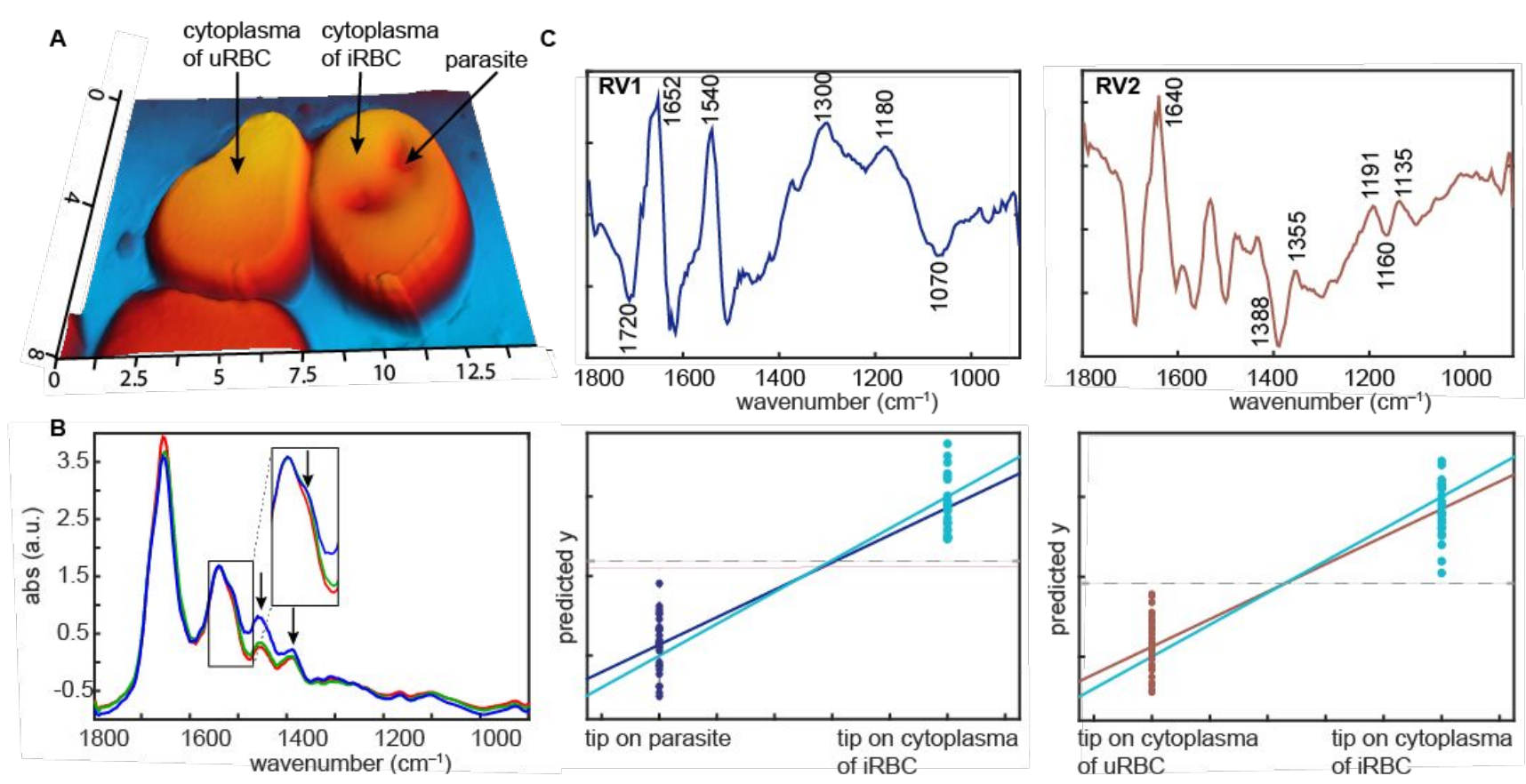

Figure 1: A: Topographic AFM map of one B. bovis-iRBC and one URBC. Arrows indicate the positions on which IR spectra were recorded. B: Averaged IR spectra (9 smoothing points, vector normalized) acquired directly on the parasite from the iRBC and on the $\mathrm{URBC}$ (red=from cytoplasm of iRBC, green=parasite, blue=uRBC). C: PLS-DA regression vectors (RV, upper panels) and 


\section{prediction plot (lower panels) showing the prediction against the position of the AFM-tip on the sample.}

The PLS-DA regression vectors (RV) shown in Figure 1C can be used to highlight differences between spectra taken from the parasite, from the cytoplasm of the iRBC and from the $\mathrm{URBC}$. The positive bands at $1652 \mathrm{~cm}^{-1}$ and $1540 \mathrm{~cm}^{-1}$ in RV1 and at $1640 \mathrm{~cm}^{-1}$ in RV2 match the amide I and amide II bands arising mainly from $\mathrm{C}=\mathrm{O}$ stretching in the amide I band and a combination of $\mathrm{N}-\mathrm{H}$ bending and $\mathrm{C}-\mathrm{N}$ stretching modes in the amide II band. ${ }^{18}$

The positive values in the carbohydrate region in RV1 might arise from alkyl group vibrations (at $1300 \mathrm{~cm}^{-1}$ ) and vibrations from propionyl and higher ester groups in carbohydrates $\left(\text { at } 1180 \mathrm{~cm}^{-1}\right)^{19}$ and demonstrate a higher absorbance from carbohydrates in the cytoplasm of the iRBC than the parasite directly. A comparison of the cytoplasm of the iRBCs and the $\mathrm{URBC}$ shows a shift in the carbohydrate composition rather than a change in the total absorbance.

The most remarkable differences between the two RVs, however, are the negative values around $1720 \mathrm{~cm}^{-1}$ to $1740 \mathrm{~cm}^{-1}$ in RV1, which are assigned to a $\mathrm{C}=0$ stretching mode from ester carbonyl modes of lipids and the negative value at $1070 \mathrm{~cm}^{-1}$, which is tentatively assigned to a $\mathrm{PO}_{2}^{-}$ stretching mode from nucleic acids.

An inspection of the averaged spectra suggests that the difference between the iRBC cytoplasm and URBCs is superior to the difference between the parasite and the iRBC cytoplasm. This corroborates the hypothesis that the parasite drastically modifies the overall chemical composition of the iRBC by utilization of cellular components such as carbohydrates, lipids and/or proteins. To further identify phenotypic characteristics of the parasite itself, we used AFM-IR to collect images of one single $B$. bovis-iRBC. Figure $2 A$ shows the AFM topological image of a $B$. bovis-iRBC. Taking into consideration the PLS-DA RV, 14 individual wavenumber values were chosen for the collection of IR maps over the $\operatorname{iRBC}(940,970,1010,1055,1079,1106,1130,1237,1353,1457,1550,1656,1720$ and $1740 \mathrm{~cm}^{-1}$ ). These maps were registered spatially with a methodology previously described in 
the literature ${ }^{20}$ resulting in a multispectral image. The average value of the intensity maps is shown in Figure 2B. The averaged map shows less intensity on the depression of the parasite. kMCA was performed on the multispectral image to determine the wavenumber values that show a higher abundance where the parasite is positioned inside the iRBC (Figure 2C). kMCA was able to identify 4 classes. While classes $2(C 2$, red) and $3(C 3$, yellow) seem to be evenly distributed over the cell, class 1 ( $C 1$, blue) gathers along the upper edge of the cell and class 4 ( $C 4$, purple) appears in the area that was assigned to the parasite in the topographical image. Figure 2D shows the average class intensities for each of the selected wavenumber values, which contain information about the chemical composition of each class.

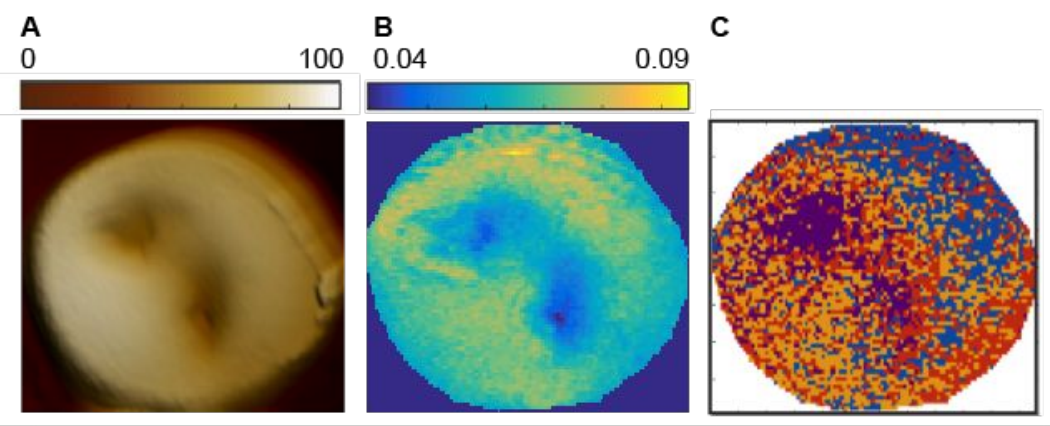

D

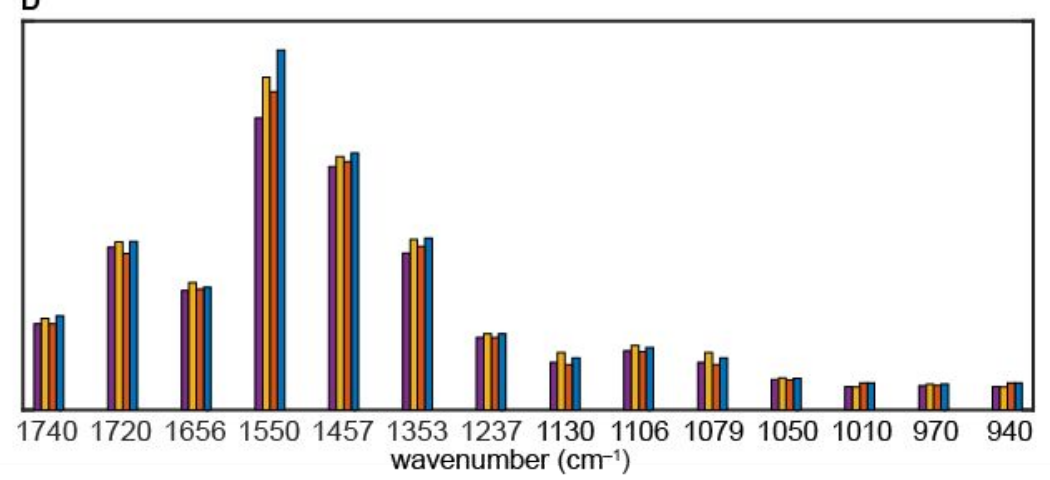

Figure 2: A: Topographic AFM image of one single B. bovis-iRBC. B: overlaid IR image. C: Classes obtained from kMCA on the multispectral IR image (class 1: blue, class 2: red, class 3: yellow and class 4: purple). D: average class intensities from kMCA on the multispectral IR image.

$\mathrm{C} 1$ has the strongest amide I $\left(1656 \mathrm{~cm}^{-1}\right)$ and amide II $\left(1550 \mathrm{~cm}^{-1}\right)$ bands. The amide II band is also strongly pronounced in C2 and C3, while C4 has the lowest value for this band.

The areas do not appear to differ in their lipid content as the ester carbonyl $\mathrm{C}=\mathrm{O}$ stretching modes that appear around $1740 \mathrm{~cm}^{-1}$ seem relative consistent, though slightly higher in C1 and C3 and 
slightly lower in C2. In the carbohydrate region, which includes vibrations from propionyl and higher ester groups in carbohydrates at $1179 \mathrm{~cm}^{-1}, 19$ the intensity is higher in $\mathrm{C} 1$ and $\mathrm{C} 2$, while lower in C3 and C4.

\section{Confocal Raman Microscopy}

Raman microspectroscopy is a method complementary to IR absorption spectroscopy. In IR spectroscopy there must exist a change in dipole moment for the molecule to be IR active, while in the case of Raman active modes the dipole moment must be induced by the laser which changes the polarizability of the molecule or functional group. Therefore, molecules that are IR active are often Raman inactive and vice versa. Hyperspectral images of iRBCs were recorded using confocal Raman microscopy. kMCA was performed on preprocessed (first derivative, mean centering) spectra of one isolated B. bovis-iRBC (Figure 3).

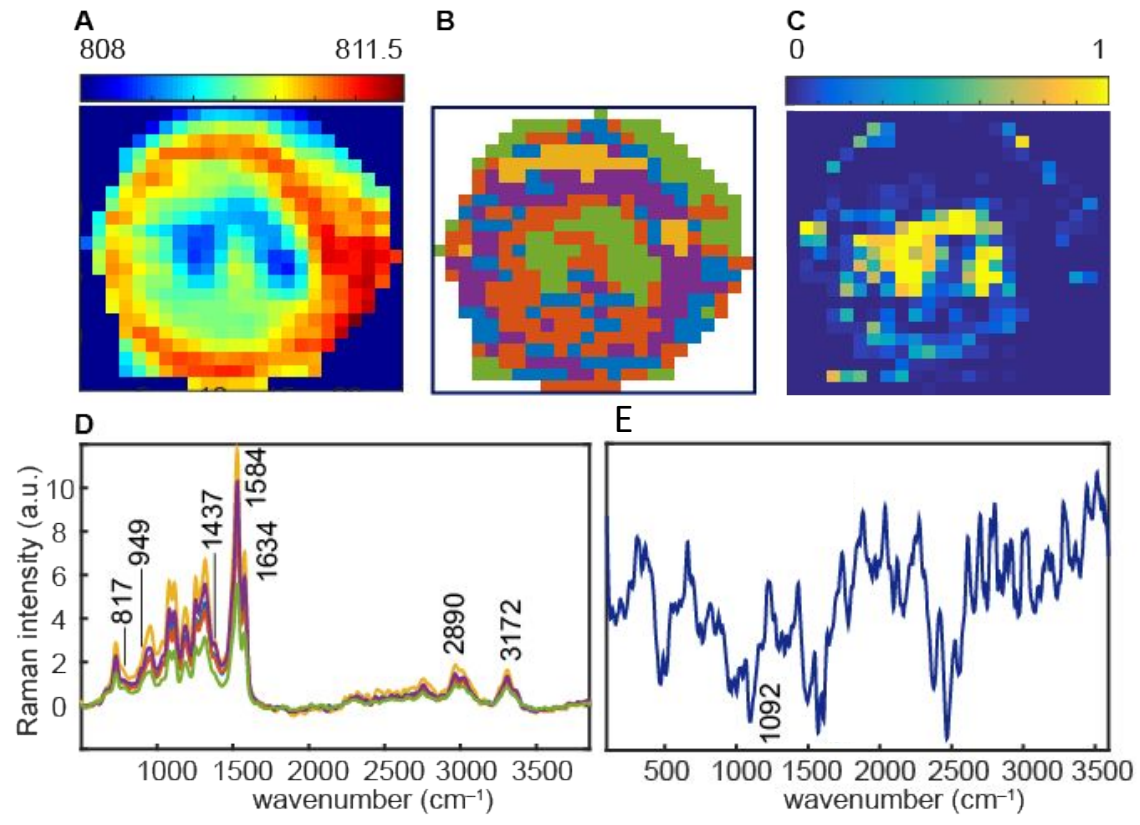

Figure 3: A: Hyperspectral Raman image based on the integrated intensity of the band at $1584 \mathrm{~cm}^{-1}$, B: kMCA cluster image, C: Probability of being parasite for each pixel calculated by a PLSDA D: integrated average spectrum of each class E: PLS-DA regression vector. Here, the positive bands arise from molecules with abundance in the parasite, while the negative bands come from molecules that are substantial in the surrounding cytoplasm. 
Figure $3 \mathrm{~A}$ shows the hyperspectral Raman image based on the integrated intensity of the band at $1584 \mathrm{~cm}^{-1}$. PLS-DA was performed over all pixels in the hyperspectral image. Classes were manually selected from the image in Figure 3A and assigned to "parasite" and "cytoplasm" (spectra recorded from cytoplasm of the iRBC but not on the parasite itself). Figure 3B shows the UHCA for a single red blood cell infected with the Babesia bovis parasite using 5 clusters. Figure $3 C$ shows the probability with which each class is present in one pixel based on a value calculated from the PLS-DA prediction scores. The yellow (high probability) area confirms the presence of the parasite in the collapsed region of the image. Figure 3D shows the averaged spectra for each cluster. C5 (green) is more abundant where the parasite is located and around the edges of the RBC. There are some marked differences between the average class spectra. C5 has a lower intensity in the protein $\left(1634 \mathrm{~cm}^{-1}\right.$ and $1584 \mathrm{~cm}^{-1}$ ) and fingerprint region (spectral region $<1500 \mathrm{~cm}^{-1}$ ) of the spectrum; while the rest classes show stronger signal intensity in the protein region with strong bands at $1634 \mathrm{~cm}^{-1}$ and 1584 $\mathrm{cm}^{-1}$, the latter being a classical heme band. ${ }^{21}$ Moreover, the parasite itself seems to have similar lipid content compared to the other classes because the averaged spectra of $\mathrm{C} 5$ have similar intensity to the other classes in the $\mathrm{CH}$ stretching region $\left(>2800 \mathrm{~cm}^{-1}\right)$, a region that is among others associated with symmetric $\mathrm{CH}_{3}$ stretching vibrations at $2929 \mathrm{~cm}^{-1}$ and asymmetric $\mathrm{CH}_{2}$ stretching vibrations at $2890 \mathrm{~cm}^{-1}$ of acyl chain lipids. ${ }^{22,21}$

\section{Detection of Babesia bovis-infected RBCs using ATR-FTIR spectroscopy}

The infrared phenotypic studies show that not only the presence of the parasite in an iRBC is observed but also principal changes in the overall chemical composition of iRBCs emerge, which give rise to changes in the vibrational spectra. ATR-FTIR spectroscopy, on the other hand, is quick and easy to use and detects changes in a bulk sample rather than in individual RBCs. Therefore, this method serves as an optimal means to develop a PoC spectroscopy-based method for B. bovis diagnosis. 
RBCs themselves show strong infrared absorbance arising from biological molecules such as membrane lipids, proteins and carbohydrates, a fact that can be easily overcome when single-cell measurements are performed. Thus, detecting a parasite in a bulk sample of RBCs means the detection of rather small changes in the composition of lipids, proteins and carbohydrates and the presence of parasitic products and DNA. To increase the sensitivity of the detection of in vitro cultured parasites in samples, we subjected RBCs to hypotonic lysis using distilled water. This causes the RBC membrane to rupture, thereby releasing the parasites and dividing the fraction into lysate and membrane fractions. Centrifugation and removal of the supernatant results in a high concentration of parasites with a reduced hemoglobin background from the red blood cell pellet and represents a truly novel approach to spectroscopic diagnosis of bovine babesiosis.

ATR-FTIR spectra were recorded using B. bovis-iRBCs diluted with uRBCs to obtain a parasitaemia of 0.25 to $1 \%$ (lower parasitaemias are shown in the SI) and compared to uRBC control. The chosen percentage matches parasitemia levels observed in infected cattle. ${ }^{23}$ B. bovis infection was predicted based on PLS-DA, which was applied to the spectra. Figure 4A shows the average ATRFTIR spectra of infected $B$. bovis-iRBCs 

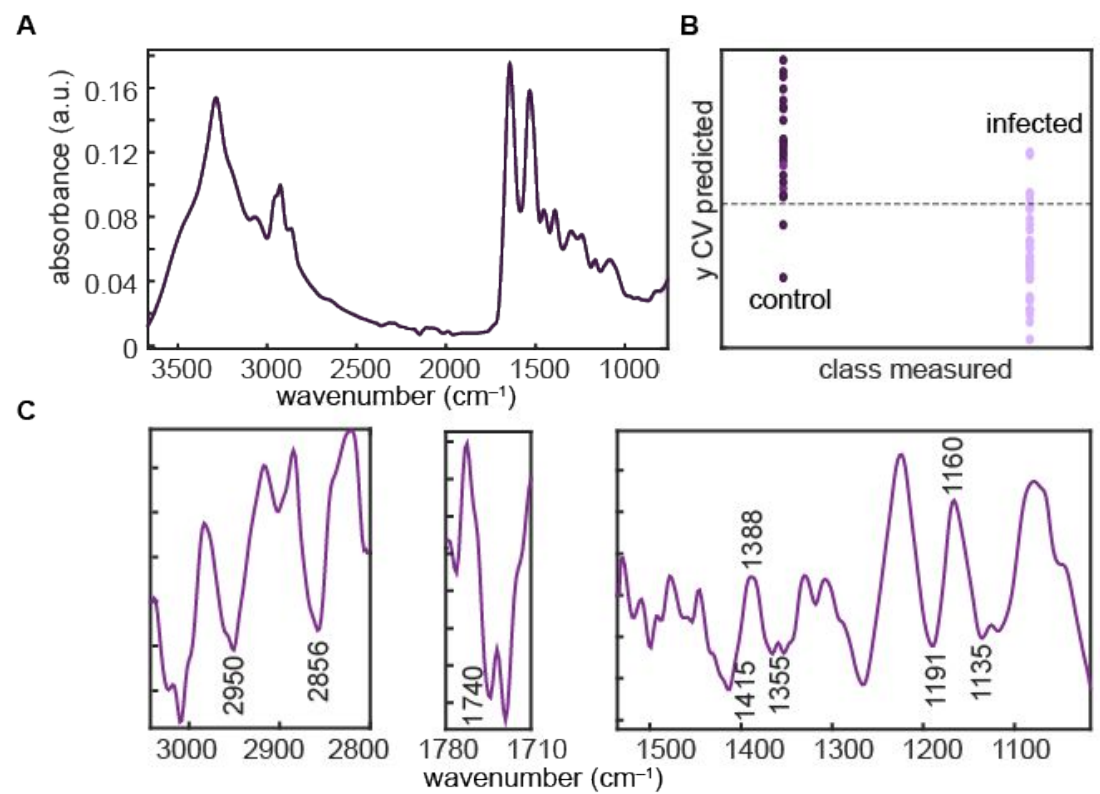

Figure 4: A: Averaged vector-normalized ATR-FTIR spectrum of infected samples. B: Predicted infection for control versus $\mathbf{0 . 2 5}$ to $\mathbf{1 \%}$ parasitemia. C: Derived PLS-DA regression vector (first derivative $\rightarrow$ second derivative) that describes the spectral features responsible for the discrimination between lysed B. bovis-iRBCs and lysed uRBCs.

Changes in the humidity of the surrounding environment cause spectral bands from water vapor to appear in the FTIR spectra. Therefore, all recorded spectra were corrected for water vapor by subtracting an independently recorded spectrum of water vapor normalized to the band between $3545 \mathrm{~cm}^{-1}$ and $3570 \mathrm{~cm}^{-1}$. Thereafter, spectra were pre-processed (spectral truncation, first derivative, SNV, scaling and mean centering) for chemometric data analysis.

The performance of the model to discriminate iRBCs from uRBCs was compared for spectra of lysed RBCs, dried onto a glass slide, to spectra of intact RBCs dried onto a glass slide (SI). PLS-DA of spectra of lysed RBCs with concentrations of $0.25 \%, 0.5 \%$ and $1 \%$ compared to uRBCs allowed for discrimination with a sensitivity of $92.0 \%$ and a specificity of $91.7 \%$ (Figure $4 \mathrm{~B}$ ). The sensitivity and specificity decreased to $77.3 \%$ and $79.2 \%$, respectively, for samples of intact RBCs at concentrations between 0.25 and $1 \%$ parasitemia. For the lysed samples, spectral regions including the $\mathrm{CH}$ stretching region (3040-2800 $\left.\mathrm{cm}^{-1}\right)$, the ester carbonyl region $\left(1800-1700 \mathrm{~cm}^{-1}\right)$ and the DNA region (1450-900 $\mathrm{cm}^{-1}$ ) were analyzed. The PLS-DA RV (Figure 4C) was processed to the second derivative 
to enable a more straightforward interpretation compared to first derivative spectra and contains information about differences in the chemical composition between the two classes.

The RV shows spectral features from stretching vibrations of $\mathrm{CH}_{3}$ groups at $2950 \mathrm{~cm}^{-1}$ and of $\mathrm{CH}_{2}$ groups at $2856 \mathrm{~cm}^{-1}$ that arise from asymmetric and symmetric vibrations, respectively. At 1766, 1745 and $1729 \mathrm{~cm}^{-1}, \mathrm{C}=\mathrm{O}$ vibrations from ester carbonyl groups from lipids or carbohydrates can be found. In the fingerprint region, the RV is in good agreement with the RVs from the AFM-IR study. The negative band at $1452 \mathrm{~cm}^{-1}$ is a $\mathrm{C}-\mathrm{H}$ deformation mode of proteins and the band at $1415 \mathrm{~cm}^{-1}$ arises from a $\mathrm{C}-\mathrm{N}$ vibration from primary amides. Both are more pronounced in the controls. Between $1388 \mathrm{~cm}^{-1}$ and $1135 \mathrm{~cm}^{-1} \mathrm{C}-\mathrm{H}$ and $\mathrm{C}-\mathrm{O}$ deformation and stretching vibrations from carbohydrates or lipids occur. ${ }^{19,24}$ These bands are partly more distinct in iRBCs and more intense in uRBCs. This suggests a shift in the carbohydrate and lipid composition rather than an increase or decrease of their amount. Biologically relevant bands are summarized in Table 2.

Table 2: Selected IR bands assigned to vibrations of biological molecules.

\begin{tabular}{|c|c|c|c|}
\hline $\begin{array}{l}\text { wavenumber (ATR- } \\
\text { FTIR) }\end{array}$ & $\begin{array}{l}\text { wavenumber } \\
\text { (AFM-IR) }\end{array}$ & Assignment & classification \\
\hline $2950 \mathrm{~cm}^{-1}$ & & $\mathrm{v}_{\mathrm{as}} \mathrm{CH}_{3}$ acyl chain lipids & iRBC/parasite \\
\hline $2856 \mathrm{~cm}^{-1}$ & & $\mathrm{v}_{\mathrm{s}} \mathrm{CH}_{2}$ acyl chain lipids & iRBC/parasite \\
\hline $1766-1729 \mathrm{~cm}^{-1}$ & $1720 \mathrm{~cm}^{-1}$ & $\begin{array}{l}\text { V } \quad \mathrm{C}=\mathrm{O} \text { (lipids, ester } \\
\text { carbonyl) }\end{array}$ & parasite \\
\hline $1452 \mathrm{~cm}^{-1}$ & $1452 \mathrm{~cm}^{-1}$ & $\delta \mathrm{C}-\mathrm{H}$ (proteins) & parasite \\
\hline $1415 \mathrm{~cm}^{-1}$ & & $\begin{array}{l}\delta C-N \quad \text { (prim. amide, } \\
\text { amide III) }\end{array}$ & iRBC \\
\hline $1388 \mathrm{~cm}^{-1}$ & $1388 \mathrm{~cm}^{-1}$ & $\delta \mathrm{C}-\mathrm{H}$ & uRBC \\
\hline $1310 \mathrm{~cm}^{-1}$ & $1300 \mathrm{~cm}^{-1}$ & alkyl groups & uRBC \\
\hline $1265 \mathrm{~cm}^{-1}$ & & $v \mathrm{C}-\mathrm{O}$ & $\mathrm{iRBC}$ \\
\hline $1224 \mathrm{~cm}^{-1}$ & & $\begin{array}{l}\text { proteins, amide III or RNA } \\
\mathrm{V}_{\mathrm{as}} \mathrm{PO}_{2}^{-}\end{array}$ & URBC \\
\hline
\end{tabular}




$\begin{array}{lllll}1191 \mathrm{~cm}^{-1} & 1191 \mathrm{~cm}^{-1} & \vee C-O & \text { iRBC } \\ 1160 \mathrm{~cm}^{-1} & 1160 \mathrm{~cm}^{-1} & \text { ester } & \text { (carbohydrates) } & \\ & & \text { groups } & \text { URBC } \\ 1135 \mathrm{~cm}^{-1} & 1135 \mathrm{~cm}^{-1} & \text { C-H skeletal vibration } & \text { iRBC }\end{array}$




\section{Discussion}

We applied spectroscopic imaging techniques to study $B$. bovis-induced phenotypic changes in parasite-iRBCs. The data obtained serve as a benchmark to establish an ATR-FTIR spectroscopybased tool for the rapid PoC diagnosis of Babesia infections.

There are some technical considerations that should be taken into account when comparing ATRFTIR and AFM-IR spectra. The penetration depth of ATR-FTIR has been studied in depth previously ${ }^{25,26}$ and is defined as the depth at which the electric field falls to $1 / \mathrm{E}$ of its value at the surface. Considering the ATR measurement of RBCs using the conditions in this study (angle of incidence and refractive index of sample and diamond), the penetration depth $(1-3 \mu \mathrm{m})$ is well above the thickness of an RBC. In addition, when the lysate of an RBC is considered, a homogenous solution is measured and the penetration depth becomes irrelevant. However, the use of AFM-IR is relatively new, and there is still not a consensus as to the actual "penetration depth" (i.e. which layers of sample below the tip contribute to the spectrum and how the contribution decays with depth) of the technique. Recent measurements of the Plasmodium spp. parasite ${ }^{20}$ have shown that the spectra contain contributions from different cell components such as hemozoin, hemoglobin or lipids. Most of these components are found below the cell membrane, indicating that the AFM-IR spectra represents to some extent the composition of internal organelles. The most pronounced $B$. bovis-induced phenotypic variation appears in the protein region $\left(1700-1500 \mathrm{~cm}^{-1}\right)$. In the topographic AFM image, the parasite manifests as depressions in the RBCs (Figure $2 \mathrm{~A}) .{ }^{14,27}$ This collapse might be explained by a reduction in the content of structural proteins on the surface of the $\mathrm{iRBC}$ around the parasite compared to the rest of the cell leading to weakening if the cytoskeleton, which is in agreement with the single point AFM-IR measurements in this study (Figure 1). Comparison of AFM-IR single point spectra taken with the AFM tip on top of the parasite, on the cytoplasm of the iRBC and on the cytoplasm of one URBC shows a lower intensity of the amide I and amide II bands in the parasite. The positive values in PLS-DA regression vector 1 (See figure 2c), 
which compares the parasite area to the cytoplasm of the iRBC show a higher occurrence of proteins in the cytoplasm than directly on the parasite.

Between the cytoplasm of iRBCs and URBCs, a shift in protein secondary structure is indicated by a shoulder that appears at $1512 \mathrm{~cm}^{-1}$ in $\mathrm{URBCs}$ that is not present in iRBCs. Furthermore, the amide I and amide II bands assigned in the PLS-DA regression vector 2 that contains information about differences in the chemical composition between iRBCs and URBCs are both shifted towards lower wavenumber values, which indicate a higher abundance of proteins with $\beta$-sheet secondary structure in iRBCs. The positive values in regression vector 2 with a slight wavenumber shift indicative of protein secondary structural changes in iRBCs compared to uRBCs. ${ }^{28}$ This is in agreement with the observations in the averaged spectra in Figure $1 \mathrm{~B}$ and can be explained by a decrease of the relative content of hemoglobin in iRBCs in favor of proteins excreted by the parasite. KMCA of the AFM-IR image identified 4 classes and shows lower intensity of the amide I and amide II bands in class 4 , which clusters around the parasite. The amide I and amide II bands in class 1 (blue, Figure 2) class 1 can be attributed to a high hemoglobin concentration in this region. The amide II band is also strong in class 2 (red, Figure 2) and class 3 (yellow, Figure 2), while class 4 (purple, Figure 2) has the lowest value, which is coherent with the assumption that less protein is present around the parasite.

kMCA of the hyperspectral Raman image identified 5 classes. In the averaged spectra of class 5 (green, Figure 3), which corresponds to the parasite, a shortfall of spectral features associated with proteins, is found. This especially evident with the $1584 \mathrm{~cm}^{-1}$ band assigned to heme.$^{21}$

PLS-DA was performed over all pixels in the image assigning one class as "parasite" and one as "cytoplasm" to further establish the spectral differences between the parasite and the cytoplasm of the iRBC. The classes were discriminated based on spectral features shown in the regression vector, which showed a lower intensity of the heme band at $1584 \mathrm{~cm}^{-1}$ in the parasite. This band arises from molecular vibrations of hemoglobin that is observed to be less abundant in the location 
of the parasite. This can be assigned to lower hemoglobin content and, analogous to the interpretation of the AFM-IR data, to a decrease in structural proteins that lead to a collapse of the cytoskeleton in the cell. This corroborates the finding that in Plasmodium sp.-infected human RBCs, the spectrin and actin junctions in the cell membrane are disrupted, which leads to dismantlement of the cytoskeleton ${ }^{15}$. This supports the view that collapse of the cell in the position of the parasite that we observed can be explained by disruption of the RBC cytoskeleton by the presence of the Babesia parasite.

Besides the lower protein content in the parasite, there seems to be a higher abundance of lipids. This is implied, on the one hand, by the stronger intensity of bands around $1720-1740 \mathrm{~cm}^{-1}$ ( $C=0$ stretching mode of triacyl esters) in the PLS-DA regression vector from AFM-IR single point measurements. On the other hand, the intensity of Raman signals in the lipid region (CH-stretching vibrations at $>2800 \mathrm{~cm}^{-1}$ ) stays approximately the same as opposed to the protein region. Cluster analysis confirms this assumption as class 5 (green, Figure 3 ) is distributed in the parasite as well as around the edges of the cell, where membrane lipids are present. The positive loadings in the PLSDA regression vector 1 from AFM-IR single point analysis in the carbohydrate region (1300 and 1180 $\mathrm{cm}^{-1}$ ) along with the higher intensity of the same band in class 1 and class 2 from the cluster analysis indicates a lower abundance of carbohydrates in the parasite.

The information from phenotypic studies was used to develop a spectroscopy-based method for $B$. bovis diagnosis. In combination with chemometric data analysis, ATR-FTIR spectroscopy allows for the discrimination between B. bovis iRBCs (0.25-1\% parasitemia) and control uRBCs. Again; a combination of spectral regions that are associated with biological molecules was considered. While protein bands were observed to have less intensity in the infected samples, which is in accordance with the findings from phenotypic imaging, a shift in the carbohydrate and lipid bands was also observed. However, including the amide I and amide II bands that are characteristic for proteins in 
the analysis, deteriorated PLS-DA results using spectral data acquired from lysed samples. This might be attributed to an irreproducible denaturation of the proteins when the sample is treated with distilled water. The PLS-DA regression vector from ATR-FTIR (Figure 4) studies is in good agreement with regression vector 2 from the AFM-IR study (Figure 1) that compares spectral features of the cytoplasm of iRBCs to spectral features of the cytoplasm of URBCs, while less agreement can be found between the regression vectors from the ATR-FTIR and the AFM-IR study where spectral features from the parasite itself were compared with those from the cytoplasm of iRBCs. The $C=0$ ester carbonyl stretching vibration from lipids appearing around 1720 to $1740 \mathrm{~cm}^{-1}$ is strongly pronounced in the parasite as found by single point measurements of a single iRBC. This finding is confirmed by the stronger signal from this band in the negative RV values from PLS-DA on ATR-FTIR data that represent the infected samples. Hence, infected samples seem to have a higher lipid content compared with uninfected samples, which might be attributed to the parasitic membrane and to possible lipid contributions from the numerous internal parasite organelles containing a membrane. This is consistent with previous studies characterizing the lipid composition of iRBCs. ${ }^{29-}$ ${ }^{31}$ The same applies for the $\mathrm{C}-\mathrm{H}$ deformation mode from proteins at $1452 \mathrm{~cm}^{-1}$, which is more intense in infected samples as seen from the ATR-FTIR RV and is correlated with the parasite in regression vector 1 from AFM-IR measurements.

The $\mathrm{C}-\mathrm{H}$ deformation mode at $1388 \mathrm{~cm}^{-1}$, the alkyl group vibrations around $1300 \mathrm{~cm}^{-1}$ and the ester group vibrations of carbohydrates at $1160 \mathrm{~cm}^{-1}$, on the other hand, show positive values in the ATRFTIR RV and account for control samples, and are characteristic for uRBCs from single point AFM-IR measurements. The $\mathrm{C}-\mathrm{O}$ stretching vibration at $1191 \mathrm{~cm}^{-1}$ and the $\mathrm{C}-\mathrm{H}$ skeletal vibration at 1135 $\mathrm{cm}^{-1}$ show negative values in the ATR-FTIR RV and account for infected samples, as well as representing the $\mathrm{URBC}$ from single point AFM-IR measurements. A direct comparison between the regression vectors (Figure S1, SI) shows the strong agreement between the ATR-FTIR RV with regression vector 2 from the AFM-IR study that compares spectral features of the cytoplasm of iRBCs 
to spectral features of the cytoplasm of uRBCs. This suggests that changes of the overall chemical composition of the RBC cytoplasm are more important for discrimination between infected and uninfected cells than spectral features of the parasites themselves.

In ATR-FTIR, the $\mathrm{C}-\mathrm{H}$ stretching region (2800 to $3100 \mathrm{~cm}^{-1}$ ) was also taken into consideration. Here, a shift in the spectral bands is possibly associated with an increase of phosphatidylcholine and decrease of sphingomyelin in B. bovis infected samples. ${ }^{29}$ Moreover, more short lipid acid chains seem exist to in the infected sample than in the uninfected control, as the $\mathrm{CH}_{2} / \mathrm{CH}_{3}$ ratio is smaller in infected samples (Table 2). ${ }^{32}$

In summary, the attribution of changes in the chemical composition to specific IR and Raman bands appears challenging. Nevertheless, taking into account the overall spectral changes that observe all features over the whole spectral range combined allows for clear assessment of $B$. bovis infection. One of the major strengths of spectroscopy as a diagnostic tool is that not only one biomarker is detected, but even small shifts in the chemical composition of positive and negative samples can be detected, when a broad spectral range is considered.

\section{Conclusions}

The application of imaging techniques provided information about changes in the chemical composition between $B$. bovis infected and uninfected samples. AFM-IR with three different tip positions (on the parasite, on the surrounding cytoplasm of the iRBC and on the $\mathrm{URBC}$ ) revealed that differences between IRBCs and URBCs are superior to the differences between the parasite and the cytoplasm of the iRBC. This is in agreement with the hypothesis that the parasite drastically modifies the overall chemical composition of the RBC by the utilization of blood components such as carbohydrates or proteins leading to a destruction of the cytoskeleton followed by a collapse of the 
iRBC. The KMCA image could be superimposed on the AFM image and shows what bands are associated with parasite and cytoplasm in an iRBC. The main difference noted was a reduction of protein in the presence of the parasite, which is not surprising given the lack of hemoglobin in the region of the parasite.

Based on the above phenotypic studies, the utility of ATR-FTIR spectroscopy for $B$. bovis diagnosis was established. As ATR-FTIR on blood samples is usually hampered by the background absorbance of blood components, blood samples were lysed thereby isolating parasite and membrane components. This increased the detection sensitivity to $92.0 \%$ for $0.25 \%$ parasitemia, which is in the physiological range of parasite levels in the blood of infected cattle ${ }^{23}$ demonstrating the possibility to translate ATR-FTIR as a simple and quick diagnostic tool in the field. A diagnostic tool that does not require specific biomarkers, but looks into changes of the overall chemical composition of a blood sample permits diagnosis without a symptomatic diagnosis. As B. bovis and B. bigemina can co-exist in a single bovine, future ATR-FTIR studies addressing the differential diagnosis among those two diseases are planned in our lab.

\section{Acknowledgements}

BRW is supported by Australian Research Council Discovery Project (DP180103484). BMC and CES are supported by grants from the Australian Research Council and The International Development Research Centre, Canada. DPG acknowledges support from the European Research Council MSCA grant (Spectro-metrics, 020-MSCA-IF-2017 Project ID:796287)

\section{Supporting Information}

Additional information on the comparison between the ATR-FTIR and AFM-IR regression vectors (PDF). 


\section{References}

(1) Bock, R.; deVos, A. J.; Molloy, J. B. Tick-borne diseases of Cattle; Australian and New Zealand Std Diag Proc, 2006, 1, 1-29.

(2) Mcleod, R.; Kristjanson, P., Economic Impact of Ticks and Tick-borne Diseases to Livestock in Africa, Asia and Australia; Australian Cent Inter Agric Res 1999, 1, 1-6.

(3) Spickler, A.R.; Factsheet:Bovine Babesiosis; Cen Food Secur Pub Health 2003 (Last Updated: 2008), 1-10. http://www.cfsph.iastate.edu/Diseaselnfo/factsheets.php

(4) Diuk-Wasser, M. A.; Vannier, E.; Krause, P. J. Coinfection by Ixodes Tick-Borne Pathogens: Ecological, Epidemiological, and Clinical Consequences; Trends Parasitol 2016, 32, 30-42.

(5) Vannier, E.; Krause, P. J. N Human babesiosis; Engl J Med 2012, 366, 2397-2407.

(6) Bock, R.; Jackson, L.; de Vos, A.; Jorgensen, W. Babesiosis of cattle; Parasitology 2004, 129 Suppl, S247-269.

(7) Griffiths, P. R.; De Haseth, J. A. Fourier Transform Infrared Spectrometry, 2 ed.; John Wiley \& Sons, Inc., 2007.

(8) Bunaciu, A. A.; Aboul-Enein, H. Y.; Fleschin, Ş. Vibrational Spectroscopy in Clinical Analysis; App Spec Rev 2015 2, 176-191.

(9) Perez Guaita, D.; Kochan, K.; Martin, M.; Andrew, D.; Richards, J.; Heraud, P.; Wood, B. Multimodal vibrational imaging of cells; Vib Spec 2017, 91 46-58.

(10) Kong, K.; Kendall, C.; Stone, N.; Notingher, I. Raman spectroscopy for medical diagnostics--From in-vitro biofluid assays to in-vivo cancer detection; Adv Drug Deliv Rev 2015, 89, 121-134.

(11) Khoshmanesh, A.; Dixon, M. W.; Kenny, S.; Tilley, L.; McNaughton, D.; Wood, B. R. Detection and quantification of early-stage malaria parasites in laboratory infected erythrocytes by attenuated total reflectance infrared spectroscopy and multivariate analysis; Anal Chem 2014, 86, 4379-4386.

(12) Jackson, L. A.; Waldron, S. J.; Weier, H. M.; Nicoll, C. L.; Cooke, B. M. Babesia bovis: culture of laboratory-adapted parasite lines and clinical isolates in a chemically defined medium; Exp Parasitol 2001, 99, 168-174.

(13) Vega, C. A.; Buening, G. M.; Rodriguez, S. D.; Carson, C. A. Cloning of in vitro propagated Babesia bigemina; Vet Parasitol 1986, 22, 223-233.

(14) Hutchings, C. L.; Li, A.; Fernandez, K. M.; Fletcher, T.; Jackson, L. A.; Molloy, J. B.; Jorgensen, W. K.; Lim, C. T.; Cooke, B. M. New insights into the altered adhesive and mechanical properties of red blood cells parasitized by Babesia bovis; Mol Microbiol 2007, 65, 1092-1105.

(15) Millholland, M. G.; Chandramohanadas, R.; Pizzarro, A.; Wehr, A.; Shi, H.; Darling, C.; Lim, C. T.; Greenbaum, D. C. The malaria parasite progressively dismantles the host erythrocyte cytoskeleton for efficient egress; Mol Cell Proteomics 2011, 10, M111 010678.

(16) Barth, A. Infrared spectroscopy of proteins; Biochim Biophys Acta 2007, 1767, 1073-1101.

(17) Shaw, R. A.; Mantsch, H. H. In Encyclopedia of AnalChemistry, Meyers, R. A., Ed.; John Wiley and Sons, 2008.

(18) Bandekar, J. Amide modes and protein conformation; Biochim Biophys Acta 1992, 1120, 123143.

(19) Tipson, S. Infrared Spectroscopy of Carbohydrates: Washington DC, 1968; Vol. 110.

(20) Perez-Guaita, D.; Kochan, K.; Batty, M.; Doerig, C.; Garcia-Bustos, J.; Espinoza, S.; McNaughton, D.; Heraud, P.; Wood, B. R. Multispectral Atomic Force Microscopy-Infrared Nano-Imaging of Malaria Infected Red Blood Cells; Anal Che. 2018, 90 (5), 3140-3148.

(21) Rygula, A.; Majzner, K.; Marzec, K. M.; Kaczor, A.; Pilarczyka, M.; Baranskaa, M. Raman spectroscopy of proteins: a review; J Raman Spec 2013, 44, 1061-1076. 
(22) Czamara, K.; Majzner, K.; Pacia, M. Z.; Kochan, K.; Kaczora, A.; Baranskaa, M. Raman spectroscopy of proteins: a review; J Raman Spec 2015, 46, 4-20.

(23) Urquhart, G. M.; Armour, J.; Duncan, J. L.; Dunn, A. M.; Jennings, F. W. In Veterinary Parasitology; Blackwell Science: Harlow, United Kingdom, 1999.

(24) Tamm, L. K.; Tatulian, S. A. Infrared spectroscopy of proteins and peptides in lipid bilayers; $Q$ Rev Biophys 1997, 30, 365-429.

(25) Gaigneaux, A.; Goormaghtigh, E. A New Dimension for Cell Identification by FTIR Spectroscopy: Depth Profiling in Attenuated Total Reflection. Analyst 2013, 138 (14), 40704075. https://doi.org/10.1039/C3AN00193H.

(26) Averett, L. A.; Griffiths, P. R.; Nishikida, K. Effective Path Length in Attenuated Total Reflection Spectroscopy. Anal. Chem. 2008, 80 (8), 3045-3049. https://doi.org/10.1021/ac7025892.

(27) Scudiero, L.; Mercado-Rojano, W. J.; Rudolph, A.; Wang, J.; Laughery, J. M.; Suarez, C. E. J Microsc 2018, 271, 84-97.

(28) Oberg, K. A.; Ruysschaert, J. M.; Goormaghtigh, E. The optimization of protein secondary structure determination with infrared and circular dichroism spectra; Eur J Biochem 2004, 271, 2937-2948.

(29) Florin-Christensen, J.; Suarez, C. E.; Florin-Christensen, M.; Hines, S. A.; McElwain, T. F.; Palmer, G. H. Phosphatidylcholine formation is the predominant lipid biosynthetic event in the hemoparasite Babesia bovis; Mol Biochem Parasitol 2000, 106, 147-156.

(30) Florin-Christensen, J.; Suarez, C. E.; Florin-Christensen, M.; Wainszelbaum, M.; Brown, W. C.; McElwain, T. F.; Palmer, G. H. A unique phospholipid organization in bovine erythrocyte membranes; Proc Natl Acad Sci USA 2001, 98, 7736-7741.

(31) Gimenez, G.; Florin-Christensen, M.; Belaunzaran, M. L.; Isola, E. L.; Suarez, C. E.; FlorinChristensen, Evidence for a relationship between bovine erythrocyte lipid membrane peculiarities and immune pressure from ruminal ciliates; J Vet Immunol Immunopathol 2007, 119, 171-179.

(32) Derenne, A.; Claessens, T.; Conus, C.; Goormaghtigh, E. In Gated Stimulated Emission Depletion Microscopy (g-STED) 2013, pp 1074-1081. 


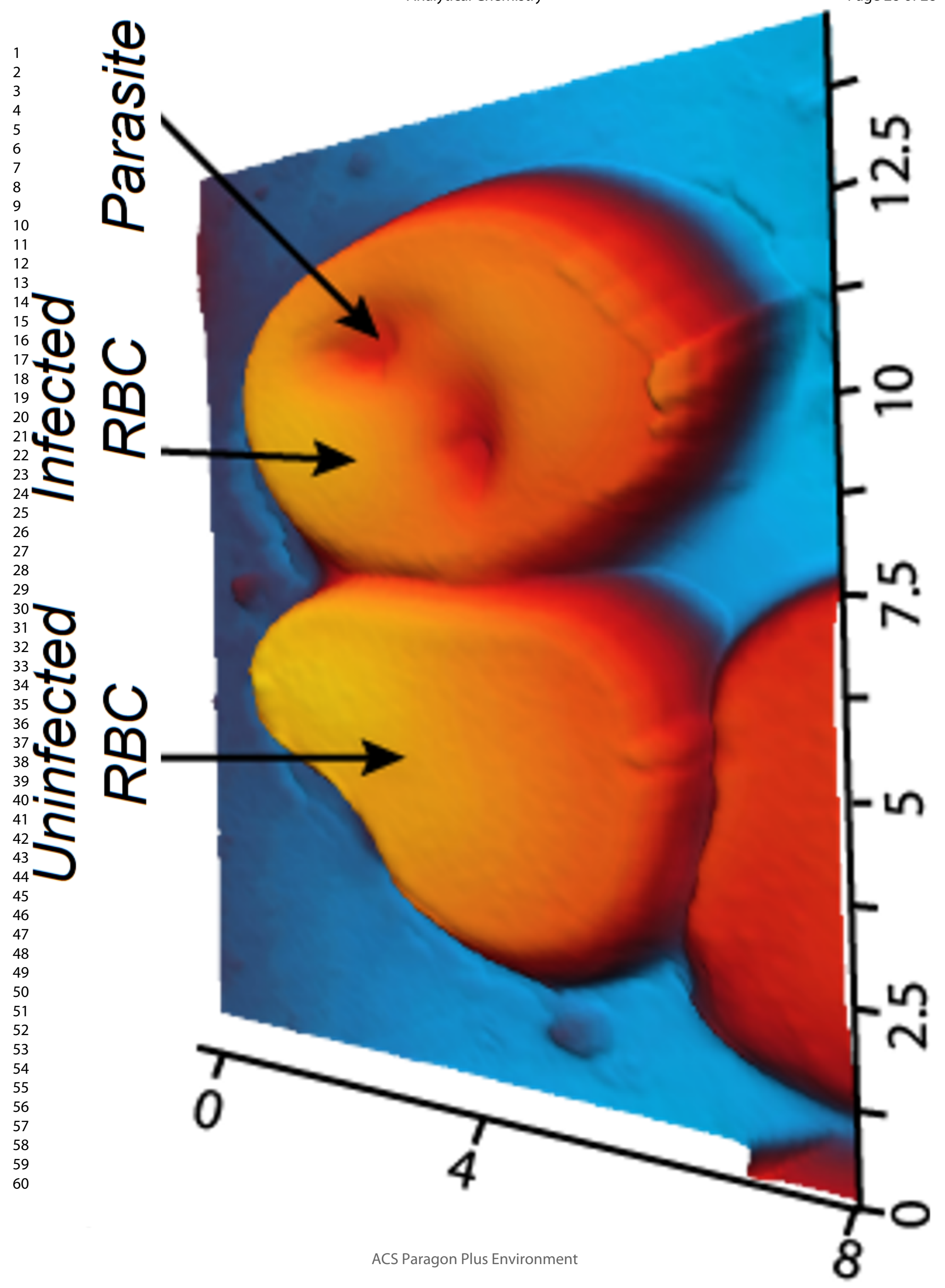

